\title{
Newly Modified Descemetopexy Method in Descemet's Membrane Detachment and Prognostic Analysis: A Systematic Review
}

\section{Zhan Shen}

Peking University Third Hospital

\section{Rongmei Peng}

Peking University Third Hospital

\section{Gege Xiao}

Peking University Third Hospital

Jing Hong ( $\square$ hongjing196401@163.com )

Peking University Third Hospital https://orcid.org/0000-0002-8079-2073

Research article

Keywords: Descemet's membrane detachment, descemetopexy, vacuum technique, detachment duration

Posted Date: August 20th, 2020

DOI: https://doi.org/10.21203/rs.3.rs-54725/v1

License: (c) This work is licensed under a Creative Commons Attribution 4.0 International License. Read Full License 


\section{Abstract}

\section{Background}

To present a newly modified descemetopexy method and investigate the most determinative factor in the prognosis of Descemet's membrane detachment (DMD).

\section{Methods}

This was a retrospective review of 37 patients (38 eyes) treated with the newly modified descemetopexy method. This surgical method was combined with the vacuum technique to pump supra-Descemet's fluid. After reviewing and screening, we enrolled 31 patients (32eyes). We evaluated outcomes using the anatomical reattachment of DM [anatomical success (AS)] and the resolution of corneal oedema [functional success (FS)]. Donor characteristics, including sex, age, the presence of diabetes, ocular history, DMD duration, DMD grade, were analysed. Patients were divided into two groups according to whether FS was achieved. Univariate and multi-factor logistic regression analyses were adopted to evaluate factors contributing to the prognosis of DMD.

Results

AS was achieved in 31 eyes (96.9\%), and FS was achieved in 21 eyes (65.6\%). The univariate analysis showed that both the DMD grade $(P=0.029)$ and $D M D$ duration $(P=0.004)$ affect the prognosis of $D M D$. The multiple logistic regression analysis indicated that the DMD duration was the dominant factor $(P=0.006)$. No significant difference was found in the DMD grade, sex, age, presence of diabetes, presence of glaucoma or DMD-originating surgery between the two groups.

\section{Conclusions}

Descemetopexy combined with the vacuum technique can be used to deal with DMD. The DMD duration is the dominant factor affecting the activity of endothelial cells and the final prognosis.

\section{Background}

Descemet's membrane detachment (DMD) has been recognized as a pathology of the posterior cornea, for close to a hundred years [1]. The first cases were described by Fuchs E in 1928[1]. The hallmark clinical feature is persistent edema of the overlying corneal stroma and epithelium. When edema involves the pupillary area, vision will be affected. DMD as a rare, potentially sight-threatening complication may occurring after various intraocular surgeries[1] [2, 3] [4] [5]. Cataract surgery is the most common, with a morbidity rate of approximately $0.044 \%$ per year[6] [7] [8] [9]. During intraocular surgeries, ingress and egress of the instrument can cause Descemet's membrane (DM) contusion and even detachment from the edge of the incision [2] [9] [10]. Corneal endothelial cells adhere to the posterior surface of DM, conducting an important dehydrating function to maintain the transparency of the cornea [2] [6]. Once 
serious detachment occurs, endothelial cells lose their dehydration function. The accumulation of fluid in corneal stromal leads to oedema, causes loss of vision[11].

The most common treatments surgical intervention known as descemetopexy (intraocular injection of an air bubble), which is performed to intervene in a serious or visual axis involving $\mathrm{DMD}[12]$. During the intracameral injection procedure with $100 \%$ air, DM is floated up by the bubble and reattached to the corneal posterior stroma. Before the bubble is completely absorbed, it can host for a while until DM adheres and will not redetach[2] [7] [13] [14] [6]. While, some surgeons have suggested that perfluoropropane (14\% C3F8) or hexafluoride $(15-20 \%$ SF6) has a longer retention time[ $[15,16]$ and may result in good outcomes. But there are more risks for endothelial toxicity and pupillary block.

Is there a way that technique can achieve good outcomes in treating DMD, avoid toxic effects and reduce the damage caused by pupillary block? In this study, we used a newly modified descemetopexy method to solve this problem.

Clinically, even if Descemet membrane was reattached, the final postoperative prognosis of the eyes still varied greatly. Some corneas regained clarity, while some suffered from corneal endothelium decompensation. There are few reports about what truly affects the prognosis. We also investigated the factors to identify the real determinative factor in the prognosis of DMD and gain insights for clinical treatment.

\section{Methods}

The aim of our study was to present a newly modified descemetopexy method and investigate the most determinative factor in the prognosis of Descemet's membrane detachment (DMD).

This was a retrospective review of patients treated with the newly modified descemetopexy method. We evaluated descemetopexy outcomes and adopted univariate and multi-factor logistic regression analyses to evaluate factors contributing to the prognosis of DMD.

Patients

This study is a retrospective review of 37 (visual axis involved) DMD patients (38 eyes) treated at Peking University Third Hospital. Institutional review board and ethics committee approval was obtained. A written and informed consent was obtained from all participants.

Patients who met the following criteria were eligible for the study: (1). Slit-lamp examination showed floating on the posterior corneal surface and corneal oedema(corresponding to the detachment area)[7]. (2).Anterior segment optical coherence tomography (AS-OCT) revealed a gap between the corneal stroma and DM[17] [18] [19]. AS-OCT (Visante Model 1000; Carl Zeiss Meditec, Inc. Dubin. CA. USA) was used from 2011 onward to aid in the diagnosis of DMD. 
We excluded patients with the following criteria: (1) Signs of a postoperative infection; (2) Had received descemetopexy in other hospitals; (3) Received a surgical treatment other than the modified descemetopexy in our hospital; (3) Incomplete original medical records.

Technique of the newly modified descemetopexy method in DMD

The detachment area, depth of DMD (the greatest distance between DM and the stroma) and the location of DM rupture were determined with a slit lamp and AS-OCT accurately before the operation.

Descemetopexy was performed under local anaesthesia. Two incisions were prepared for descemetopexy surgery(Instrument: Vitrectomy knife, straight, 20Gauge)(Supplementary video 1):

Incision no. 1: The "air injected incision" was made at the limbal site where the cornea was relatively compact and opposite to DM rupture.

Incision no. 2: The "puncture incision" was made at the point of the cornea where detachment was the most obvious (the visual axis area was avoided) (Fig. 1; Fig. 1.A). This operation was recommended to be performed under the slit lamp if possible.

$100 \%$ sterile air was injected by a flushing needle $(0.45 \mathrm{~mm}$ diameter) through incision no. 1 into the anterior chamber (AC) smoothly and continuously. DM was reset with an equally expanded bubble and attached to the posterior surface of the stroma. Meanwhile, supra-Descemet's fluid slowly flowed out from incision no. 2 (Fig. 1; Fig. 1.B). When the air bubble almost completely filled the AC, we use a cotton swab to lightly press the incision and pulled out the needle to ensure that the bubbles will not overflow. We determined the situation of Descemet's membrane and the amount of supra-Descemet's fluid under the slit lamp. After that, we use a flushing needle $(0.45 \mathrm{~mm}$ diameter) draw residual supra-Descemet's fluid at the edge of incision no.2. If there was too much fluid, we might extent the needle into the interlayer through the "puncture incision", draw residual supra-Descemet's fluid while sliding. (Fig. 1; Fig. 1.C). Finally, we supplemented sterile air until the air bubbles filled the $A C$ and ensured that the intraocular pressure was within the normal range. DM was supported by both the bubble's buoyancy and the vacuum's attraction interlayer (Fig. 1; Fig. 1.D).

Patients were asked to maintain a supine position for at least 4 hours to ensure that the bubble support for the DM to continue adhering [20] [20]. We measured the intraocular pressure 2 hours postoperatively (with I-care). When intraocular pressure showed higher than $40 \mathrm{mmHg}$, we released about $40 \%$ residual air to make sure of pupil margin exposure to prevent ocular hypertension caused by a pupil block.

Preoperative and postoperative clinical features

The preoperative ocular evaluation included a previous history of ocular disease; DMD-originating surgery; DMD duration time (the time interval between DMD and air descemetopexy); and the grade of DMD (graded under a slit lamp cornea; moderate DMD involving 1/4-1/2 of the cornea; and severe DMD involving $>1 / 2$ of the cornea or with central involvement) $[21][7,22]$. 
The postoperative clinical features included the intraocular pressure (IOP), corneal condition, bubble content in the AC,postoperative corneal thickness and corneal edema grades (grade 0 (transparent); grade 1 (minimal haze, details of iris and pupil distinct); grade 2 (mild haze, iris and pupil detectable); grade 3 (moderate haze, iris and pupil hardly visible); grade 4 (opaque, iris and pupil not discernable)) [23].

The descemetopexy outcome evaluation included anatomical success (AS) and functional success (FS), defined as follows: AS: DM is attached to the posterior surface of the corneal stroma with or without slight corneal oedema ( $\leq$ grade 2 ) [7, 16]. It is observed using a slit lamp or AS-OCT. (AS is chosen as the main criterion to evaluate the effect of this newly modified descemetopexy method.) ; FS: With the seamless reattachment of DM, the cornea completely becomes transparent without oedema under a slit lamp and OCT [7, 16] (Supplementary Fig. 1). (FS is one of the main criteria used to evaluate late postoperative outcomes).

\section{Statistical analysis}

The variables examined included sex, age, the presence of diabetes, the presence of glaucoma, DMDoriginating surgery, DMD duration (days), and DMD grade.

We carried out univariate analyses of the associations between each factor and the ideal ultimate prognosis (FS) by $\chi 2$ test. $P \llbracket 0.05$ was considered statistically significant. A multiple logistic regression analysis using the backward stepwise (Wald) model was adopted for all of the associated significant factors identified in the univariate analyses $(P<0.05)$. Some variables with great clinical value but no significant were still included in the multiadjusted models as confounding factors. A two-sided $P$ value less than 0.05 was considered statistically significant and was calculated in a backward stepwise (Wald) multivariate logistic regression model. All analyses were conducted using IBM SPSS Statistics 24.0 (International Business Machines Corp).

\section{Results}

The records of 37 patients (38 eyes) were reviewed. After screening, 31 patients (32 eyes) were enrolled in the study(Figure 2).

Supplementary Table 1 lists the patients' preoperative demographics, clinical characteristics, and characteristics of the patients after descemetopexy treatment: The mean age of the patients was 72.0 years (range: $43-91$ years), the male to female ratio was 19:12, and the ratio of right to left eye involvement was 15:17). Diabetes were noted in 7 (22.5\%) patients. Glaucoma was noted in $5(16.1 \%)$ patients. As for the DMD-originating surgery, 24 patients [25 eyes (78\%)] was secondary to cataract surgery, 7 patients (12\%) was secondary to anti-glaucoma surgery. After modified descemetopexy treatment, 31 (96.9\%) eyes achieved AS, including corneal edema grade0 11 eyes; grade1 14 eyes; grade2 5eyes. 21 (65.6\%) eyes achieved FS. 11 eyes (24.4\%) showed a nonideal late prognosis. Among these eyes, redetached DM occurred in 7 eyes, and irreversible severe corneal oedema occurred in 4 eyes. 
Table 1 shows the prognosis of DMD over time. The mean time interval from cataract surgery to intervention was 38 days. 21 eyes had a detachment duration fewer than 30 days, 18 (85.7\%) eyes achieved FS; 11 eyes had a detachment duration over 30 days, 3 eyes achieved FS (27.3\%). In view of the significant decrease in the rate of eyes that achieved FS at 30 days, we used 30 days as the boundary to explore whether 'detachment time of more than 30 days' is one of the dominant factors affecting the late prognosis of DMD.

The univariate analysis revealed that the grade of DMD $(P=0.029)$ and detachment duration $(P=0.004)$ showed statistical significance (Table 2). No differences were found with respect to sex, the presence of diabetes, the presence of glaucoma, or DMD-originating surgery. In the multivariate analysis, we still included sex, age, and DMD-originating surgery considering their great clinical significance. The final outcome of the multiple logistic regression analysis revealed detachment duration ( $\leq 30$ days) as a significant independent factor for the prognosis of DMD (OR, 15.441; P, 0.006). No significant difference was found in the DMD grade, detachment time, sex, age, presence of diabetes, presence of glaucoma or DMD-originating surgery between the two groups. 
Table 2

Characteristics of Patients with DMD treated with Descemetopexy and Univariate Analysis of Possible Factors Involved in the Prognosis of DMD

\begin{tabular}{|c|c|c|c|c|c|}
\hline Variable & & $\begin{array}{l}\text { Success cases } \\
(\%)\end{array}$ & $\begin{array}{l}\text { Fail cases } \\
\text { (\%) }\end{array}$ & $X^{2}$ & $\mathbf{P}$ \\
\hline \multirow[t]{2}{*}{ Sex } & Male & $12(57.1)$ & $7(63.6)$ & 0 & 1 \\
\hline & F-Male & $9(42.8)$ & $4(36.4)$ & & \\
\hline \multirow[t]{2}{*}{ Diabetes } & No & $17(81.0)$ & $8(72.7)$ & 0.007 & 0.933 \\
\hline & Yes & $4(19.0)$ & $3(27.2)$ & & \\
\hline \multirow[t]{2}{*}{ Glaucoma } & No & 16 (76.19) & $11(100)$ & 1.561 & 0.212 \\
\hline & Yes & $5(23.80)$ & $0(0)$ & & \\
\hline \multirow[t]{2}{*}{ Incentive surgery } & $\begin{array}{l}\text { Phaco + } \\
\text { IOL }\end{array}$ & $19(90.5)$ & $6(54.5)$ & 3.553 & 0.059 \\
\hline & Others & $2(9.5)$ & $5(45.4)$ & & \\
\hline \multirow[t]{2}{*}{ Preoperation } & No & $16(76.2)$ & $6(54.5)$ & 0.728 & 0.394 \\
\hline & Yes & $5(23.8)$ & $5(45.4)$ & & \\
\hline \multirow[t]{4}{*}{ Grade of DMD } & Mild & $2(9.5)$ & $0(0)$ & 8.033 & $0.029 *$ \\
\hline & Moderate & 7 (33.3) & 2 (18.2) & & \\
\hline & Severe & $12(57.1)$ & $5(45.5)$ & & \\
\hline & Whole & $0(0)$ & $4(36.3)$ & & \\
\hline \multirow{2}{*}{$\begin{array}{l}\text { Detachment duration time ( } \leq 30 \\
\text { days) }\end{array}$} & No & 18 (85.7) & $3(27.3)$ & 8.492 & $0.004^{\star}$ \\
\hline & Yes & $3(14.3)$ & 8 (72.7) & & \\
\hline \multicolumn{6}{|c|}{ *P value significant at $\leq 0.05$; DMD: Descemet's membrane detachment; } \\
\hline \multicolumn{6}{|c|}{ F-Male = Female; Phaco + IOL = Phacoemulsification and intraocular lens implantation; } \\
\hline \multicolumn{6}{|c|}{$\begin{array}{l}\text { Grade of DMD }[\text { Mild }(\text { detachment area }<1 / 4) \text { Moderate }(1 / 4<\text { detachment area }<1 / 2) \text { Severe } \\
\text { (detachment area }>1 / 2) \text { Whole }=\text { Whole detachment] }\end{array}$} \\
\hline
\end{tabular}

\section{Discussion}

Prognostic Determinants

Based on the final outcome of the multiple logistic regression analysis, we considered detachment duration time should be the primary factor of the ultimate prognosis of DMD. The multiple logistic regression analysis revealed detachment duration ( $\leq 30$ days) as the only significant independent factor 
for the prognosis of DMD: the longer detachment duration was, the worse the prognosis. Compared to the detachment duration, other factors do not have an equal impact on the prognosis of DMD.

DM can be well restored and perfectly healed because its nutrient fountain is aqueous humour. But If the DM layer (with endothelial cells) floats in the circulating aqueous humour for too long, corneal endothelial cells will shed, and the activity of DM will be weakened (Fig. 3). The flexibility of DM may also decrease over time. In addition, the smooth posterior corneal stroma will be scarred due to the direct impaction of the aqueous humour cycle, which also contributes to the difficulty in DM reattachment[24].

Annamalai Odayappan reported that the timing of the intervention did not influence the reattachment rate in his series of 112 patients [7]. This finding may be because their detachment duration's interval length was not long enough to show statistical significance. In our study, most patients (29/31) experienced DMD after undergoing cataract surgeries in other hospitals; therefore, more time passed before they arrived at our department and were diagnosed with DMD. Thus, the detachment duration's interval length was long enough (from 8 hours to 150 days). In Annamalai Odayappan's study, the median detachment duration time was 4 days, with a reattachment rate of nearly $80 \%$. In our study, the mean detachment duration was 38 days (the longest detachment duration time ever reported), with a $65.6 \%$ FS rate. indirectly proves that the longer the detachment duration time is, the worse endothelial function is and the worse the final prognosis.

We also found that when the detachment duration time was more than 30 days, the reattachment rate decreased significantly. If detachment duration was as long as 90 days, there was no hope to achieve FS by descemetopexy. The multiple logistic regression analysis also revealed detachment duration as the only significant independent factor for the prognosis of DMD. Therefore, we determined that detachment duration is the most important factor in the final prognosis of DMD, and we suggest an immediate intervention for DMD.

The grade of DMD also influence the final prognosis of DMD. Mild DMD can be better reset with a bubble compared to severe DMD. Large area detachment often accompanies a great distance between DM and the stroma. To reset DM to the posterior stroma, more force and pressure need to be applied. According to Z. Ismail, different detachment areas may cause different local flow rates of the aqueous humour cycle. In some mild cases, the shear force exerted by the flow on DM tends to help DM remain taut. While in serious cases such as complete DMD, it is difficult to calculate the flow rates around the detached DM. It is also difficult to determine whether the flow is still effectual $[25,26]$. In addition, the larger the detachment area is, the more likely it is to create slight folds when reset treatment is adopted, which also contributes to the undesirable prognosis of DMD.

However, in the subsequent multiple logistic regression analysis, the grade of DMD was excluded, so compared to the detachment time, it has relatively little effect on the prognosis of DMD. These results are similar to those obtained by Chameen Samarawickrama in 2015[26]. 
In the results of our study, other factors, including the surgical reason, presence of diabetes, presence of glaucoma and age, showed no significant differences. We do not think their impact on the prognosis of DMD is as significant as we expected.

The modified descemetopexy

The most common treatments surgical intervention known as descemetopexy (intraocular injection of an air bubble), which is performed to intervene in a serious or visual axis involving DMD. The choice of material for descemetopexy has been a research focus in recent years. Many authors advocate that descemetopexy with $100 \%$ air has a lower risk for endothelial toxicity and a pupillary block. However, it may be less effective because of rapid systemic absorption. Once supra-Descemet's fluid exists, DM redetachment would ensue immediately after air absorption. To date, the success rates of descemetopexy with $100 \%$ air have been reported range from only $80-95 \%[16,27][7,28]$.

Compared with $100 \%$ sterile air, longer acting gas mixtures have better stability and retention times[15, 16], which may lead to better outcomes. However, longer acting gas mixtures had a higher risk for endothelial toxicity and a pupillary block even central retinal artery occlusion (CRAO) or branch retinal arterial occlusion (BRAO) [29]. To date, the success rates of descemetopexy with longer acting gas mixtures have been reported to range from $71.64-95 \%[16,30]$. There is still no consensus on the choice of materials.

In our study, our surgical method achieved ideal results and the AS rate (96.9\%) was the highest ever reported. we determined that the retention time of bubbles in the AC was not the key to success; surgical methods are more important. Our newly modified descemetopexy method combines the advantages of both $100 \%$ air and long-acting gas mixtures. On the one hand, we chose $100 \%$ sterile air as the material for descemetopexy to lower the risk for endothelial toxicity. The rapid absorption rate of air can greatly shorten the time of pupillary block and reduce its risk. On the other hand, we added the "vacuum technique" to solve the problem of the presence of supra-Descemet fluid and guaranteed the seamless reattachment of DM.

we summarize the newly modified descemetopexy in four tips as follows:

(1) The "air injected incision" should be made at the limbal site where the cornea is relatively compact and opposite to DM rupture.

(2) The "puncture incision" should be made at the point of the cornea where detachment is the most obvious (avoiding the visual axis area) to release the aqueous humour interlayer[31].

(3) The "vacuum technique" was added to remove supra-Descemet's hu mour interlayer, which may hinder the apposition of DM to the stroma completely. Remember to proceed slowly and gently with the suction step. Excessive suction can cause DM folds and endothelial damage. 
(4) Finally, supplement sterile air until the air bubbles fully fill the AC and ensure the intraocular pressure is within the normal range. In this way, DM can be reattached to the corneal posterior stroma seamlessly and be fixed by both the bubble's buoyancy and the vacuum's attraction interlayer.

There are some limitations to this study. It is a retrospective study and has a relatively small sample size; the prognosis needed to be evaluated in a larger patient group. It cannot be ruled out that increasing the suction step may cause a slight disturbance in the aqueous humour and affect FS. Further research is needed.

\section{Conclusions}

Descemetopexy combined with the vacuum technique leads to ideal anatomical outcomes. We suggest that the "suction step" be added to improve the AS rate. Detachment duration time should be the primary factor of the FS rate of DMD. The tardier the treatment is, the more severe the endothelial damage is and the worse the outcomes. Surgeons should be more prudent when recognizing this sight-threatening complication and should adopt an intervention immediately. Physicians are also expected to inform patients with DMD symptoms before intraocular surgery is adopted so that patients can be well informed and able to detect symptoms and seek timely medical treatment.

\section{Abbreviations}

AC

Anterior chamber

AS

Anatomical success

AS-OCT

Anterior segment optical coherence tomography

BRAO

Branch retinal arterial occlusion

CRAO

Central retinal artery occlusion

DM

Descemet's membrane

DMD

Descemet's membrane detachment

F-Male

Female

FS

Functional success

IOP

Intraocular pressure 
Phaco + IOL

Phacoemulsification and intraocular lens implantation

\section{Declarations}

Ethics approval and consent to participate

This study was approved by the Ethics Committee of the Peking University Third Hospital and followed the tenets of the Declaration of Helsinki. A written and informed consent was obtained from all participants.

Consent for publication

Not applicable.

Availability of data and materials

The datasets used and analysed during the current study are available from the corresponding author on reasonable request.

Competing interests

The authors declare that there is no competing interest.

Funding

This research project was supported by the National Natural Science Foundation of China (Grant No. 81970768).

Authors' contributions

PRM \& XGG provided clinical information and figures. SZ wrote the manuscript. HJ wrote and revised the manuscript. All authors read and approved the final manuscript.

Acknowledgements

Not applicable.

\section{References}

1. Samuels B. Detachment of Descemet's Membrane. Trans Am Ophthalmol Soc. 1928;26:427-37.

2. Graves SS, Storb R: Developments and Translational Relevance for the Canine Hematopoietic Cell Transplantation Preclinical Model. Vet Comp Oncol 2020. 
3. Slenter IJM, Hermans H, Ensink JM, Willems DS, Veraa S, Grinwis GCM, Boeve MH. Clinical, ultrasonographic, and histopathologic findings in seven horses with Descemet's membrane detachment: A case series. Vet Ophthalmol 2019.

4. Pujari A, Swamy DR, Chaniyara MH, Sharma N: Intraoperative Descemet's membrane detachment in a case of phacomorphic glaucoma. BMJ Case Rep 2018, 2018.

5. Fu Y, Zhou W, Li W, Lin X, Dai Q. Late-onset descemet membrane detachment and corneal decompensation after laser peripheral iridotomy: A case report. Med (Baltim). 2018;97(10):e0083.

6. Yuksel E, Cubuk MO, Ceylanoglu KS. Intracameral air injection after completion of phacoemulsification cataract surgery: Evaluation of corneal incisions with optical coherence tomography. J Curr Ophthalmol. 2019;31(2):142-9.

7. Odayappan A, Shivananda N, Ramakrishnan S, Krishnan T, Nachiappan S, Krishnamurthy S. A retrospective study on the incidence of post-cataract surgery Descemet's membrane detachment and outcome of air descemetopexy. Br J Ophthalmol. 2018;102(2):182-6.

8. Chow VW, Agarwal T, Vajpayee RB, Jhanji V. Update on diagnosis and management of Descemet's membrane detachment. Curr Opin Ophthalmol. 2013;24(4):356-61.

9. Hirabayashi KE, Mark D, Lau J, Lin CC. Descemet Stripping Only for a Chronic Descemet Detachment After Cataract Surgery. Cornea. 2020;39(3):379-81.

10. Izquierdo Villavicencio JC, Mejias Smith JA, Canola Ramirez LA, Agudelo Arbelaez N. Rubio Lastra B: Hemorrhagic Descemet Membrane Detachment during Ab Interno Canaloplasty. Case Rep Ophthalmol Med. 2019;2019:3653954.

11. Ye H, Lu Y. Corneal Bullous Epithelial Detachment in Diabetic Cataract Surgery. Optom Vis Sci. 2015;92(7):e161-4.

12. Wyatt H, Ghosh J. Reposition of Descemet's membrane after cataract extraction. A case report. Br J Ophthalmol. 1969;53(4):267-9.

13. Chaurasia S, Ramappa M, Garg P. Outcomes of air descemetopexy for Descemet membrane detachment after cataract surgery. J Cataract Refract Surg. 2012;38(7):1134-9.

14. Sharma S, Fernandes M. Descemet stripping automated endothelial keratoplasty: An alternate surgical modality for Descemet's membrane detachment following hydrops in keratoglobus. Indian $\mathrm{J}$ Ophthalmol. 2020;68(3):513-4.

15. Datar S, Kelkar A, Jain AK, Kelkar J, Kelkar S, Gandhi P, Shah R, Kedia R. Repeat Descemetopexy after Descemet's Membrane Detachment following Phacoemulsification. Case Rep Ophthalmol. 2014;5(2):203-6.

16. Garg J, Mathur U, Acharya MC, Chauhan L. Outcomes of Descemetopexy with Isoexpansile Perfluoropropane after Cataract Surgery. J Ophthalmic Vis Res. 2016;11(2):168-73.

17. AlZaid A, AIDarrab A, Malik R, Owaidhah O. Ocular coherence tomography for the diagnosis of Descemet's detachment after deep sclerectomy and resolution after intracameral air injection. Am $\mathrm{J}$ Ophthalmol Case Rep. 2019;15:100509. 
18. Yoon HY, Kim HS, Hwang HB. Guided intracameral air injection based on 3D reconstructed anterior optical coherence tomography images in iatrogenic Descemet's membrane detachment: Case report. Med (Baltim). 2018;97(43):e13003.

19. Dua HS, Sinha R, D'Souza S, Potgieter F, Ross A, Kenawy M, Scott I, Said DG. 'Descemet's membrane detachment': A novel concept in diagnosis and classification. Am J Ophthalmol 2020.

20. Jun S, Choi KY, Cho YJ. Importance of Head Position after Gas Tamponade in Bilateral Descemet's Membrane Detachment Following Cataract Surgery. Korean J Ophthalmol. 2020;34(2):173-5.

21. Guo P, Pan Y, Zhang Y, Tighe S, Zhu Y, Li M, Shen X, Lin B, Pan B, Liu X, et al. Study on the classification of Descemet membrane detachment after cataract surgery with AS-OCT. Int J Med Sci. 2018;15(11):1092-7.

22. Gujar P, Bhasin P, Bhasin P. Bilateral Descemet's membrane detachment after uneventful phacoemulsification in a case of diabetes mellitus. Indian J Ophthalmol. 2013;61(1):28-9.

23. Ulas B, Altan-Yaycioglu R, Bal N. Comparison of the inhibitory effect of different doses of subconjunctival bevacizumab application in an experimental model of corneal neovascularization. Int J Ophthalmol. 2018;11(7):1090-5.

24. Morishige N, Chikama T, Yamada N, Takahashi N, Morita Y, Nishida T, Sonoda KH. Effect of preoperative duration of stromal edema in bullous keratopathy on early visual acuity after endothelial keratoplasty. J Cataract Refract Surg. 2012;38(2):303-8.

25. Ismail Z, Fitt AD, Please CP. A fluid mechanical explanation of the spontaneous reattachment of a previously detached Descemet membrane. Math Med Biol. 2013;30(4):339-55.

26. Rao A, Watson S. Possible role of aqueous humour dynamics in Descemet's membrane detachment resolution. Clin Exp Ophthalmol. 2017;45(8):834-6.

27. Ti SE, Chee SP, Tan DT, Yang YN, Shuang SL. Descemet membrane detachment after phacoemulsification surgery: risk factors and success of air bubble tamponade. Cornea. 2013;32(4):454-9.

28. Jain R, Murthy SI, Basu S, Ali MH, Sangwan VS. Anatomic and visual outcomes of descemetopexy in post-cataract surgery descemet's membrane detachment. Ophthalmology. 2013;120(7):1366-72.

29. Meena AK, Ghodke BR, Parmar GS. Central retinal artery occlusion after Descemet membrane reposition by intracameral air. A case report. Eur J Ophthalmo/ 2019:1120672119870740.

30. Chaurasia S, Ramappa M, Rao HL. Descemet Membrane Detachment in a Child With Anterior Megalophthalmos Managed Using Intracameral Perflouropropane (C3F8) Gas Injection. Cornea. 2015;34(11):1516-8.

31. Weng Y, Ren YP, Zhang L, Huang XD, Shen-Tu XC. An alternative technique for Descemet's membrane detachment following phacoemulsification: case report and review of literature. BMC Ophthalmol. 2017;17(1):109.

\section{Figures}


A

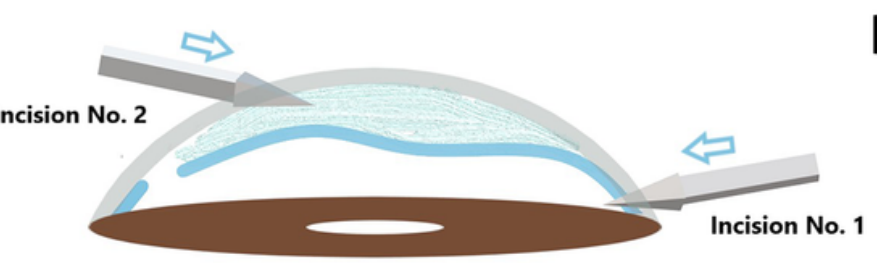

$\mathrm{C}$

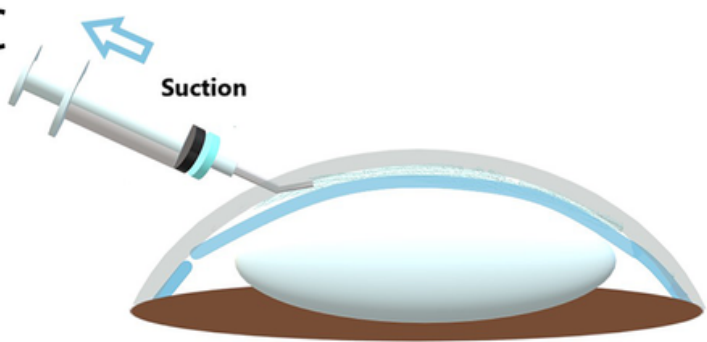

B

Outflow

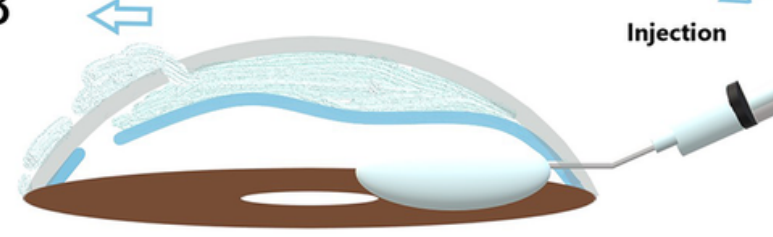

D

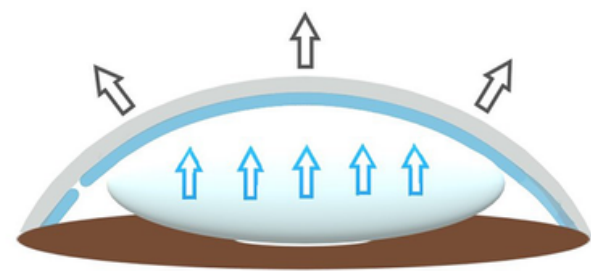

\section{Figure 1}

Technique of descemetopexy A shows the positions of the incisions. B shows the air injection. $C$ shows the vacuum step. D shows DM supported by both the bubble's buoyancy and the vacuum's attraction interlayer.

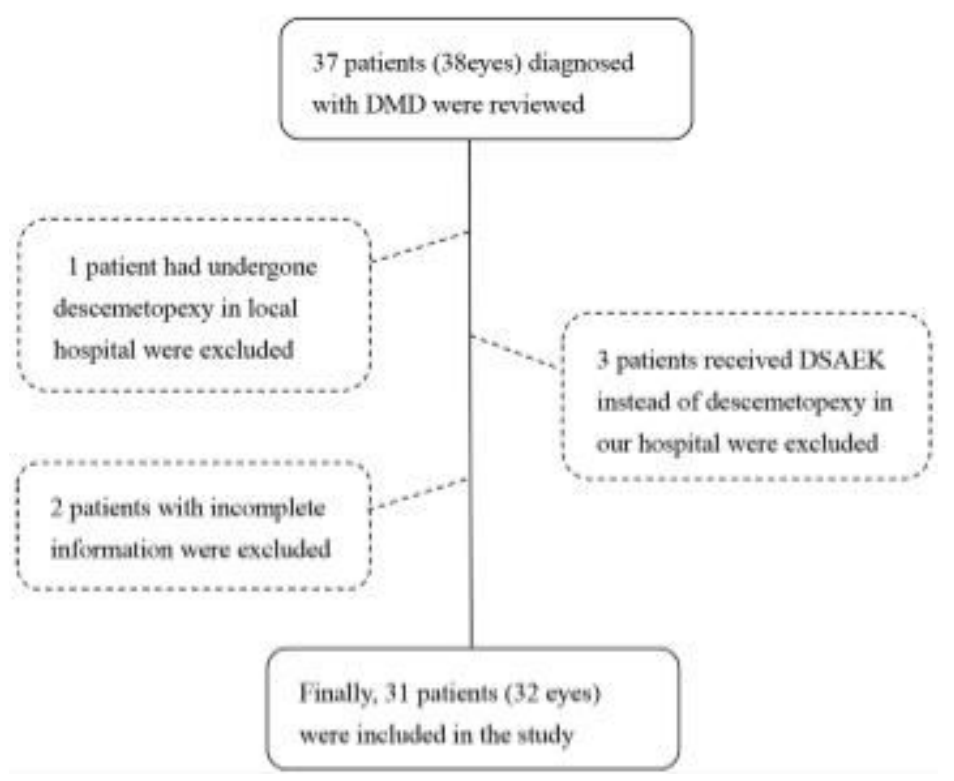

Figure 2

Flow diagram of patient selection Shows the flow diagram of patient selection 


\section{A}
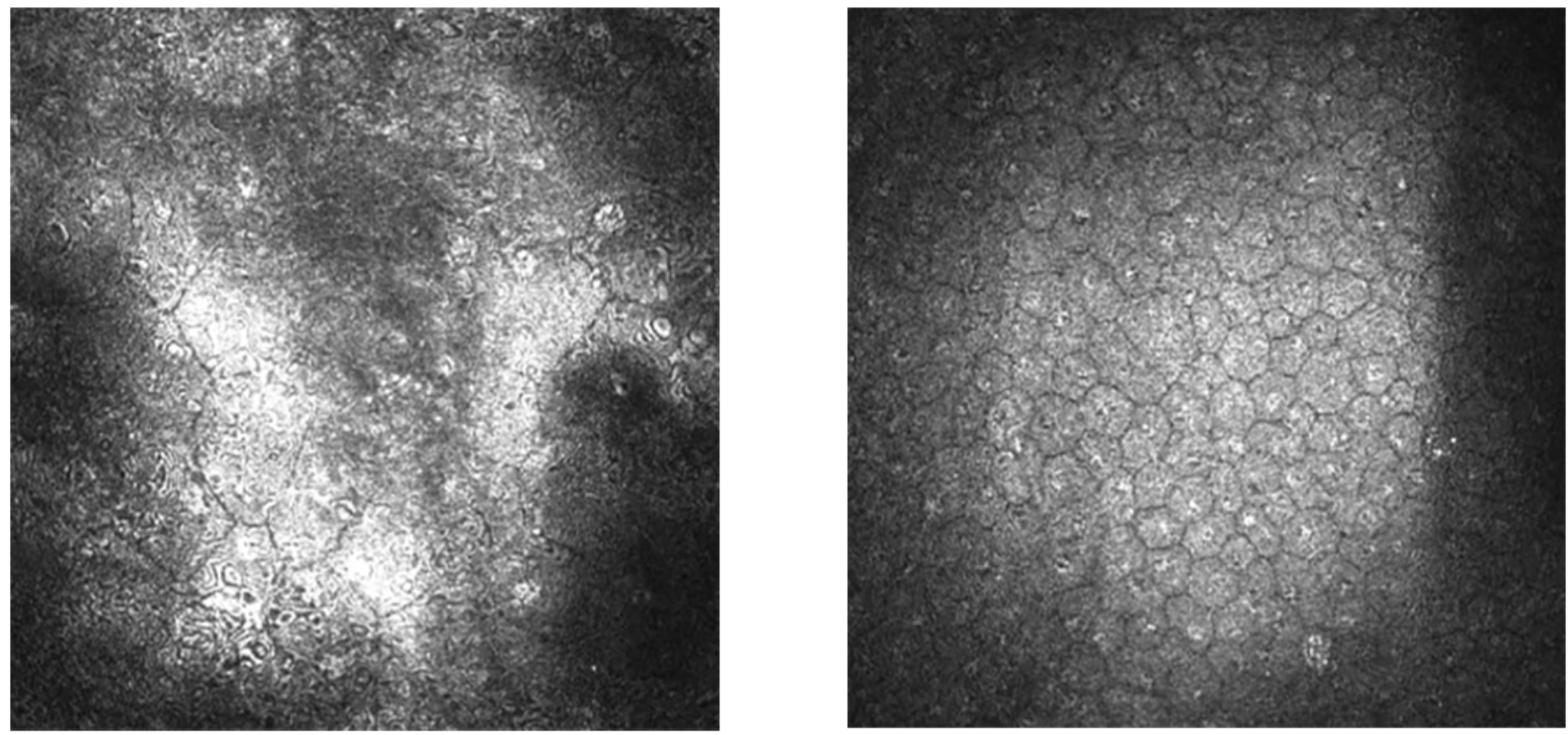

\section{Figure 3}

The endothelial conditions of patients A shows the endothelial conditions of patient no. 18 (D-duration=7 days) before descemetopexy. B shows the endothelial conditions of patient no. 3 (D-duration=150 days) before descemetopexy. The figure indicates that a long detachment duration can cause a decrease in the ECC.

\section{Supplementary Files}

This is a list of supplementary files associated with this preprint. Click to download.

- SupplementaryTable1CharacteristicsofPatients.doc

- SupplementaryFigure1Comparisonbeforeandafteroperation.jpg

- Supplementaryvideo1Techniqueofdescemetopexy.mp4 